Article

\title{
High-Precision Spectral Decomposition Method Based on VMD/CWT/FWEO for Hydrocarbon Detection in Tight Sandstone Gas Reservoirs
}

\author{
Hui Chen ${ }^{1, *}$ (D), Dan $\mathrm{Xu}^{1}{ }^{1}$, Xinyue Zhou ${ }^{1}$, Ying $\mathrm{Hu}^{1,2}$ (D) and Ke Guo ${ }^{1}$ \\ 1 Geomathematics Key Laboratory of Sichuan Province, Chengdu University of Technology, Chengdu 610059, \\ China; Dan-pp@foxmail.com (D.X.); ffgzxy@foxmail.com (X.Z.); huannahy@hotmail.com (Y.H.); \\ guoke@cdut.edu.cn (K.G.) \\ 2 Postdoctoral Station of Geophysics, Chengdu University of Technology, Chengdu 610059, China \\ * Correspondence: huichencdut@hotmail.com; Tel.: +86-288-407-3610
}

Received: 16 June 2017; Accepted: 19 July 2017; Published: 21 July 2017

\begin{abstract}
Seismic time-frequency analysis methods can be used for hydrocarbon detection because of the phenomena of energy and abnormal attenuation of frequency when the seismic waves travel across reservoirs. A high-resolution method based on variational mode decomposition (VMD), continuous-wavelet transform (CWT) and frequency-weighted energy operator (FWEO) is proposed for hydrocarbon detection in tight sandstone gas reservoirs. VMD can decompose seismic signals into a set of intrinsic mode functions (IMF) in the frequency domain. In order to avoid meaningful frequency loss, the CWT method is used to obtain the time-frequency spectra of the selected IMFs. The energy separation algorithm based on FWEO can improve the resolution of time-frequency spectra and highlight abnormal energy, which is applied to track the instantaneous energy in the time-frequency spectra. The difference between the high-frequency section and low-frequency section acquired by applying the proposed method is utilized to detect hydrocarbons. Applications using the model and field data further demonstrate that the proposed method can effectively detect hydrocarbons in tight sandstone reservoirs, with good anti-noise performance. The newly-proposed method can be used as an analysis tool to detect hydrocarbons.
\end{abstract}

Keywords: variational mode decomposition; frequency-weighted energy operator; instantaneous energy; hydrocarbon detection; tight sandstone reservoirs

\section{Introduction}

Tight sandstone gas reservoirs are widespread throughout the world and play an important part in gas exploitation [1,2]. However, it is difficult to detect hydrocarbons in tight sandstone gas reservoirs due to their deep burial depth, compactness, heterogeneities, and weak seismic response, in addition to the minimal differences between reservoirs and non-reservoirs [3-6]. Seismic attenuation is usually separated into attenuation connected to scattering effects and attenuation corresponding to stratigraphic absorption $[7,8]$. Subsurface rocks usually form a porous medium filled with fluid [9]. Waves induced by fluid flow can lead to stratum absorption, which is the main causation of attenuation of seismic waves [10,11]. When seismic waves propagate through reservoirs, high-frequency energy waves decay more rapidly than low-frequency waves, which may lead to high-frequency energy loss and only conserve strong low-frequency energy [9,12-14]. Some theoretical and practical studies have been carried out to verify this phenomenon by many scholars $[9,15-18]$. There are two main methods for revealing seismic attenuation associated with hydrocarbons, which can be classified as: (1) methods based on low-frequency information, such as low-frequency shadow analysis $[18,19]$ as well as the method of observing the difference between high-frequency and low-frequency energy [20,21]; and 
(2) methods based on frequency attenuation attributes, such as energy absorption analysis (EAA) [9,22]. Time-frequency analysis is an important tool for hydrocarbon detection by revealing the variation of frequency and energy with time $[17,23]$. Many scholars have successfully applied time-frequency analysis methods to extract instantaneous attributes for hydrocarbon detection [18,24-27].

Empirical mode decomposition (EMD) is a popular time-frequency decomposition method, which was proposed by Huang et al. [28]. The EMD method can adaptively decompose the original signals into a group of intrinsic mode functions (IMFs) in the temporal domain, with each IMF being a narrow-band signal. This method has already been successfully applied to seismic signal processing and has proven to show high time-frequency resolution in instantaneous spectral analysis [29]. However, the EMD method has several disadvantages, including its weak foundations in mathematics [30,31], end effect [32,33], and the mode-mixing problem [33,34]. Therefore, in order to overcome these problems of the EMD, ensemble empirical mode decomposition (EEMD) [32] and complete ensemble empirical mode decomposition (CEEMD) [34] are proposed. Nevertheless, the two algorithms, which are both noise-assisted analysis methods, result in the IMFs containing some noise and redundant information regarding modes. In 2014, Dragomiretskiy and Zosso proposed the variational mode decomposition (VMD) to decompose the original signals into a limited number of band-limited IMFs, each mode of which is almost compact around a corresponding center frequency. Thus, the VMD method can solve the mode-mixing problem [35] and decrease redundant modes [36]. Moreover, as the essence of VMD is an adaptive Wiener filter, the VMD method has high stability under the conditions with noise $[35,37,38]$ and can reduce residual noise in modes [35,36].

Instantaneous energy is an important seismic attribute and can be used to determine the occurrence time of amplitude anomalies $[17,39,40]$. In 1990, based on the Teager energy operator, Kaiser proposed the Teager-Kaiser energy operator (TKEO) to track the instantaneous energy of signals [41]. Furthermore, some scholars have successfully combined the time-frequency analysis methods and have confirmed that TK can track the instantaneous energy of signals for hydrocarbon detection in reservoirs $[16,17,19]$. However, the instantaneous energy values obtained by TK operators have some negative values without practical physical meaning [42]. In 2014, Toole et al. proposed a non-negative, frequency-weighted energy operator (FWEO) as a way to calculate the instantaneous energy of signals by simply combining the filtering to weight higher-frequency components with the time-varying envelop of the signals. Therefore, the FWEO method has a better performance in assessing the instantaneous energy compared to TK and is insensitive to noise [42,43].

In order to obtain the exact time-frequency distribution, the mapping operation from the time-scale domain to the time-frequency domain can be performed in CWT processing. Therefore, the selection of the scale plays an important role in the frequency distribution. For broadband non-stationary signals, a larger range of scale values and discrete intervals can result in the loss of some frequency components and influence the precision of frequency calculations [22]. Thus, it is necessary to divide broadband non-stationary seismic signals into a group of narrowband signals. Furthermore, the CWT of narrowband signals can avoid the loss of some frequency components caused by direct CWT analysis for seismic signals [22]. Based on all the above studies, a new algorithm (VMD/CWT/FWEO) is proposed in this paper for hydrocarbon detection in tight sandstone reservoirs. Firstly, the VMD/CWT method is applied to acquire a high-resolution time-frequency representation. We selected appropriate IMF components among a group number of IMFs by VMD, with the selected IMFs required to contain abnormal frequency bands of seismic signals. The CWT method was applied in the selected IMF sections to obtain high-precision time-frequency spectra. Following this, we extended the FWEO method from the time domain to the time-frequency domain, taking advantage of the method to track the instantaneous energy in the time-frequency spectra. Finally, we utilized the difference between the high-frequency and low-frequency information, which are extracted from the instantaneous energy spectra, to detect hydrocarbons. 


\section{Theories and Methods}

\subsection{Time-Frequency Decomposition Method Based on VMD/CWT}

Variational mode decomposition (VMD) is an adaptive and non-recursive decomposition method which can rapidly decompose an input signal into a discrete number of narrow-band sub-signals (intrinsic mode functions; IMFs) with specific sparsity properties in the spectral domain when reproducing the input. Each mode is mostly compact around a center frequency. The VMD method is a constrained variational optimization problem that can be written as:

$$
\min _{\left\{u_{k}\right\},\left\{w_{k}\right\}}\left\{\sum_{k}\left\|\partial_{t}\left[\left(\delta(t)+\frac{j}{\pi t}\right) * u_{k}(t)\right] e^{-j w_{k} t}\right\|_{2}^{2}\right\} \quad \text { s.t. } \sum_{k} u_{k}=x(t)
$$

where $*$ denotes the multiply operation; $\partial_{t}$ is norm of the gradient, $x(t)$ is the input signal to be decomposed; $\partial(t)$ is the Dirac function; $u_{k}$ represents the kth mode; and $w_{k}$ denotes the center frequency of the kth mode. There are many different ways to solve the reconstruction constraint. In order to render the variational problem unconstrained, Dragomiretskiy and Zosso introduced the augmented Lagrangian $L$ based on a quadratic penalty term and Lagrangian multipliers. In addition, $L$ is defined as described in previous studies [44]:

$$
L\left(\left\{u_{k}\right\},\left\{\omega_{k}\right\}, \lambda\right):=\alpha \sum_{k}\left\|\partial_{t}\left[\left(\delta(t)+\frac{j}{\pi t}\right) * u_{k}(t)\right] e^{-j \omega_{k} t}\right\|_{2}^{2}+\left\|x(t)-\sum_{k} u_{k}(t)\right\|+\left\langle\lambda(t), x(t)-\sum_{k} u_{k}(t)\right\rangle
$$

where $\alpha$ is a balancing parameter of the data-fidelity constraint.

The alternate direction method of multipliers (ADMM) [45] approach can be applied to solve Equation (2). Following this, each mode and its corresponding center frequency in the spectral domain can be obtained by:

$$
\begin{gathered}
\hat{u}_{k}^{n+1}(\omega)=\frac{\hat{x}(\omega)-\sum_{i \neq k} \hat{u}_{i}(\omega)+\frac{\hat{\lambda}(\omega)}{2}}{1+2 \alpha(\omega-\omega)^{2}} \\
\omega_{k}^{n+1}=\frac{\int_{0}^{\infty} \omega\left|\hat{u}_{k}(\omega)\right|^{2} d \omega}{\int_{0}^{\infty}\left|\hat{u}_{k}(\omega)\right|^{2} d \omega}
\end{gathered}
$$

where $\hat{x}(\omega), \hat{u}_{i}(\omega), \hat{\lambda}(\omega)$, and $\hat{u}_{k}^{n+1}(\omega)$ are the Fourier transform of $x(t), u_{i}(t), \lambda(t)$, and $u_{k}^{n+1}(t)$, respectively, and $n$ represents iterations. More importantly, Equation (3) is clearly identified as a Wiener filter of the current residual, so that the VMD method has good anti-noise performance [37].

Following this, we can obtain a high-resolution time-frequency distribution by applying the continuous wavelet transform (CWT) in the principal IMF selected from all IMFs. We name this time-frequency analysis method "VMD/CWT".

The CWT is an excellent local time-frequency analysis method, which uses a time-varying window to describe the local characteristics of a signal [22]. The CWT of a signal is defined as in a previous study [46]:

$$
W T(a, \tau)=\left\langle x(t), \psi_{a, \tau}(t)\right\rangle=\frac{1}{\sqrt{a}} \int x(t) \bar{\psi}\left(\frac{t-\tau}{a}\right) d t
$$

where $\operatorname{WT}(a, \tau)$ is the time-scale distribution of the signal; and $\psi_{a, \tau}(t)$ is mother wavelet function. The latter function is defined as:

$$
\psi_{a, \tau}(t)=\frac{1}{\sqrt{a}} \psi\left(\frac{t-\tau}{a}\right)
$$

where $\bar{\psi}$ is the conjugate form of $\psi ; a, \tau$ and $t$ are the scale parameter, time shift parameter, and time parameter, respectively.

However, different mother wavelets have different resolutions of CWT with the same signal [47]. In this paper, we adopted the Morlet wavelet as the mother wavelet. 


\subsection{Frequency-Weighted Energy Operator}

Instantaneous energy is often calculated by the amplitude squared or the envelope of the non-linear signal. Instantaneous energy is irrelevant to frequency. In 2014, Toole et al. introduced frequency information to calculate instantaneous energy by simply combining filtering to weight higher-frequency components with the time-varying envelope of the signals [42]. The new energy operator, named the frequency-weighted energy operator (FWEO), is defined as:

$$
E[x(t)]=|x(t)+j H[x(t)]|^{2}=x^{2}(t)+H[x(t)]^{2}
$$

where $x(t)$ is a signal; $x(t)$ is the first derivative of $x(t)$; and $H[\cdot]$ is the Hilbert transformation.

By making use of the central-finite difference method, we calculated that:

$$
x(n)=[x(n+1)-x(n-1)] / 2
$$

The discretization formula of the FWEO is given as:

$$
E[x(n)]=\frac{1}{4}\left[x^{2}(n+1)+x^{2}(n-1)+h^{2}(n+1)+h^{2}(n-1)\right]+\frac{1}{2}[x(n+1) x(n-1)+h(n+1) h(n-1)]
$$

where $x(n)$ is the discrete form of signal $x(t)$; and $h(n)$ is Hilbert transformation of the discrete signal $x(n)$.

Although the FWEO is similar to the TKEO, the FWEO has advantages, such as being non-negative, having noise robustness, and so on.

\section{3. $V M D / C W T / F W E O$ Method}

In this paper, a high-precision seismic time-frequency analysis method (VMD/CWT/FWEO) is proposed for hydrocarbon detection in tight sandstone reservoirs. Firstly, a high-resolution time-frequency distribution is obtained by VMD/CWT. Using the VMD method, we gain a finite number of IMF components, each of which is a narrow-band signal and has different frequency components. Following this, we select appropriate IMF components from these IMFs, with the selected IMFs required to contain the main frequency information of seismic signals. After this, the CWT method is applied in the selected IMF sections to obtain high-precision time-frequency spectra. Secondly, based on the superiority of FWEO and imitating the method of FWEO in tracking the instantaneous energy of 1D signals in the time domain, every frequency component in the time-frequency spectrum based on VMD/CWT is considered as a single signal in the time domains. FWEO is utilized to calculate the instantaneous energy for every single-frequency signal, while instantaneous spectra can be gained through lining up the instantaneous energy of every single-frequency signal. Finally, we extract the high-frequency and low-frequency information from the instantaneous spectrum obtained by $\mathrm{VMD} / \mathrm{CWT} / \mathrm{FWEO}$ and compare the difference in energy between them to detect hydrocarbons in tight sandstone reservoirs. It is noted that the method of choosing high and low frequencies is important. In practical processing, we apply VMD/CWT to some seismic signals of an original seismic section to obtain the time-frequency spectrum for observing the dominant frequency range of the seismic section. We then use the EAA to compare the energy attenuation characteristics between through-well signals and other signals. Combining the dominant frequency band and attenuation range of the signal, we can select appropriate high and low frequencies. The main procedure of the VMD/CWT/FWEO method is shown in the flowchart in Figure 1.

The VMD/CWT/FWEO method has four major advantages:

1. A more precise time-frequency distribution of seismic signals can be obtained by the VMD/CWT method.

2. The CWT is applied to the narrow-band IMFs, which can avoid the loss of some frequency components caused by direct CWT analysis for seismic signals [22]. 
3. The FWEO method not only improves the resolution of the time-frequency spectrum based on $\mathrm{VMD} / \mathrm{CWT}$, but also highlights abnormal energy and frequency on the time-frequency spectrum.

4. Owing to the noise robustness of the VMD and FWEO, the proposed method is universal and can be applied in the field seismic data.

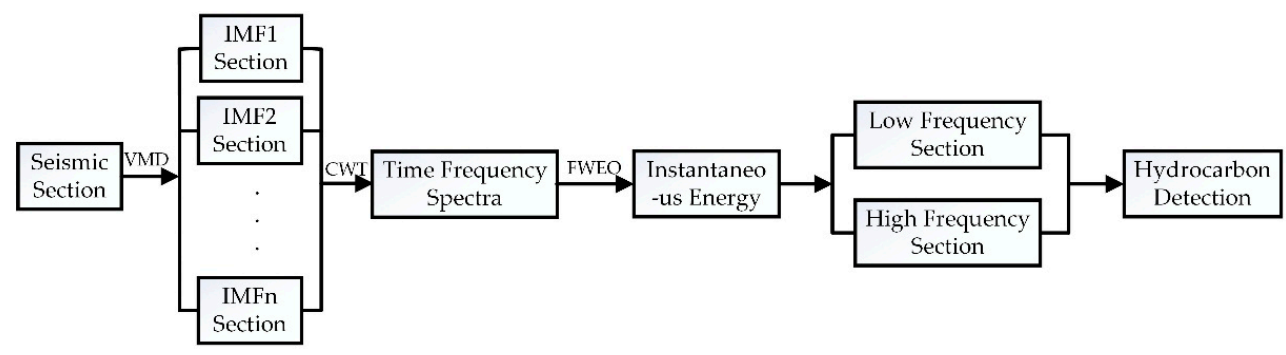

Figure 1. A flowchart of the VMD/CWT/FWEO method.

\section{Synthetic Signal}

In this section, we demonstrate the superiority of the FWEO and VMD/CWT/FWEO by taking a $1 D$ synthetic signal as an example. The synthetic signal (Figure $2 b$ ) is created by summing five distinct sub-signals (Figure 2a) as follows:

$$
\begin{aligned}
& \text { sig1 }=1.2 \cos (4 \pi t) \\
& \text { sig2 }=0.4 \sin (34 \pi t) \\
& \text { sig3 }=\left\{\begin{array}{l}
0 \text { if } t<0.5 \\
0.3 \cos (56 \pi t) \text { otherwise } \\
\text { sig4 }
\end{array}=\left\{\begin{array}{l}
0.3 \cos (80 \pi t) \text { if } t<0.5 \\
0 \text { otherwise }
\end{array}\right.\right.
\end{aligned}
$$

where $t \in[01]$. In this, sig3 and sig4 have a sharp transition between two constant frequencies. Sig5 is a seismic record simulated by an attenuated Ricker wavelet. The dominant frequency of the wavelet is $80 \mathrm{~Hz}$ and the attenuation factor $(\mathrm{Q})$ is 60 . The synthetic signal is given by the equation:

$$
\text { signal }=\operatorname{sig} 1+\operatorname{sig} 2+\operatorname{sig} 3+\operatorname{sig} 4+\operatorname{sig} 5
$$

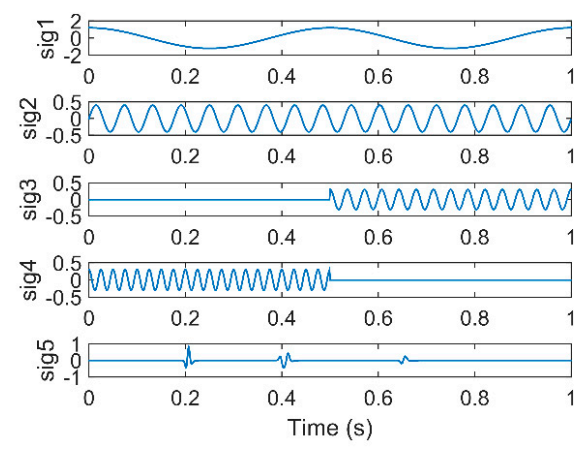

(a)

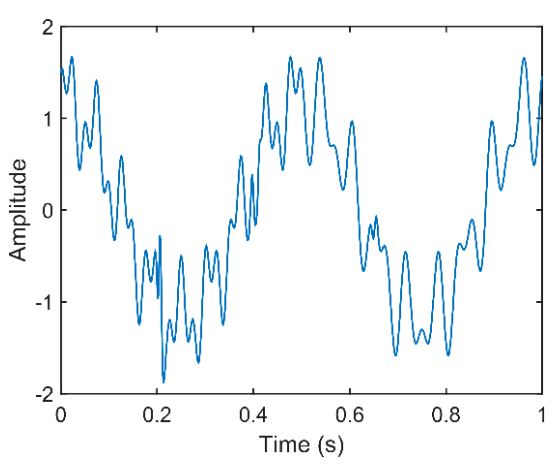

(b)

Figure 2. (a) Sub-signals; and (b) synthetic signal. 


\subsection{Performance Test of the FWEO}

TKEO is popular with many researchers for tracking the instantaneous energy and has been frequently applied in seismic signal spectral analysis. Here, we test the superiority of the FWEO compared to the TKEO.

Firstly, we obtain the instantaneous energy of the 1D synthetic signal by using the TKEO and FWEO method in sequence. The calculated results are denoted by the blue curve and purple curve, respectively, in Figure 3. It can be clearly observed that the instantaneous energy values acquired by the TKEO method have some negative values without physical meaning. However, the FWEO can not only successfully highlight the non-negative attributes, but also calculate the instantaneous energy more precisely, as indicated by the black dotted rectangle area. Therefore, the FWEO method is more reliable and promising for assessing the instantaneous energy of signals compared to the TKEO method.

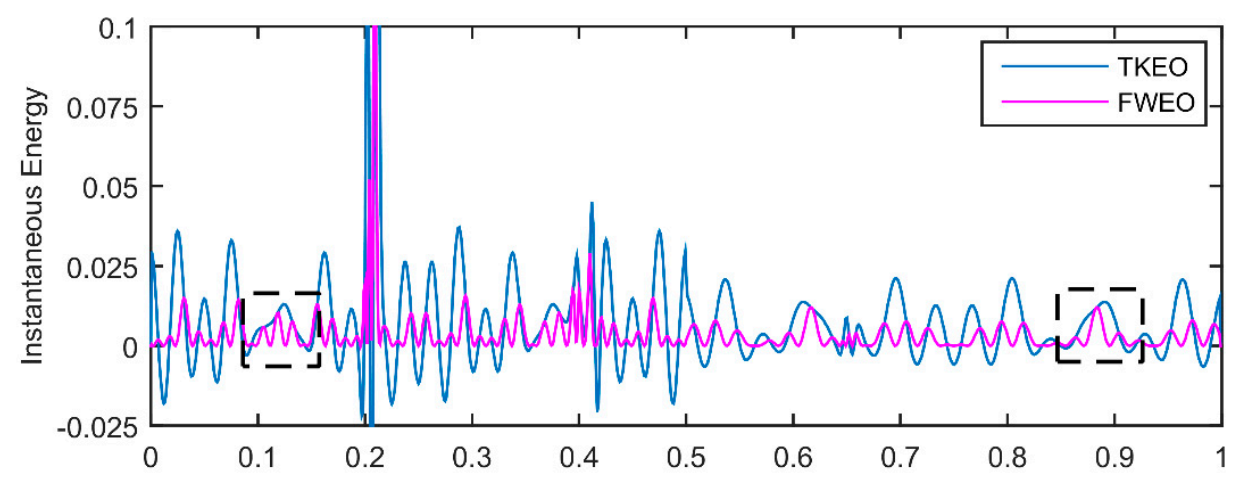

Figure 3. Instantaneous energy of the synthetic signal.

Following this, FWEO and TK are utilized to calculate instantaneous energy for every frequency component of time-frequency spectra, allowing for the instantaneous spectra to be obtained. Figure $4 \mathrm{a}$ gives the results based on TKEO, while Figure $4 \mathrm{~b}$ is the absolute values of those found in Figure $4 \mathrm{a}$. Figure $4 \mathrm{c}$ shows the instantaneous energy results based on FWEO. We easily found that there are many negative values around $2 \mathrm{~Hz}$ in Figure $4 \mathrm{a}$. Although there are no negative values in Figure $4 \mathrm{~b}$ by the operation of absolute value, the operation has no physical basis and generates some false instantaneous information. However, the end-effect problem appearing in Figure $4 \mathrm{~b}$ is resolved in Figure $4 \mathrm{c}$ by using the FWEO method, with the energy of the time-frequency spectrum in Figure 4c being more concentrated. Hence, the FWEO method can improve the time-frequency focusing property of the time-frequency spectrum to track the instantaneous energy more effectively and accurately.

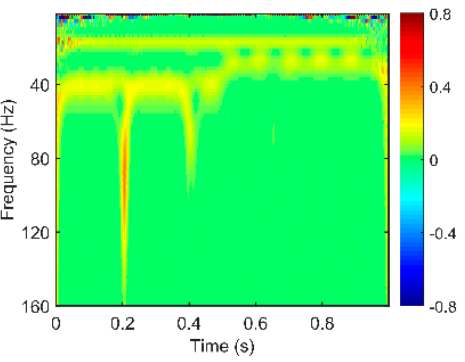

(a)

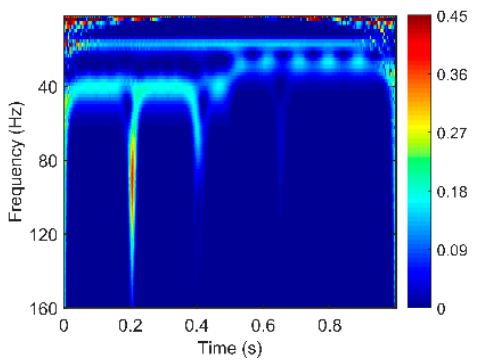

(b)

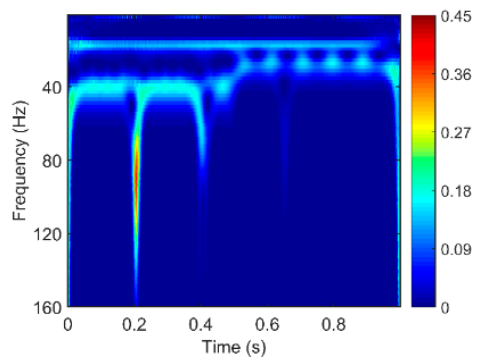

(c)

Figure 4. Instantaneous energy sections obtained based on: (a) the original TK; (b) the absolute value of the original TK; and (c) the FWEO. 


\subsection{Performance Test of the VMD/CWT/FWEO Method}

In this section, in order to demonstrate the VMD/CWT/FWEO method is a better way to calculate instantaneous energy, we compare it with the CWT/FWEO method. Figure 5a,b, respectively, give the instantaneous energy section of the 1D synthetic signal using the two different methods. It can be seen that the energy in Figure $5 b$ is more concentrated and has a higher continuity compared with Figure 5a. Furthermore, the dominant frequency and occurrence time of different sub-signals are shown in Figure 5b. For the seismic record (Sig5), the information with regards to its three wavelet forms are shown in the red dotted rectangle area. The first wavelet appeared at around $0.2 \mathrm{~s}$ with a dominant frequency of $80 \mathrm{~Hz}$. The second wavelet appeared at around $0.4 \mathrm{~s}$ with a dominant frequency of $70 \mathrm{~Hz}$. The third wavelet appeared at around $0.65 \mathrm{~s}$ with a dominant frequency of $50 \mathrm{~Hz}$. The frequency and energy attenuation between the three wavelets are clearly shown. Therefore, compared with the CWT/FWEO method, the proposed method shows a higher resolution and is an effective tool to track the instantaneous energy.

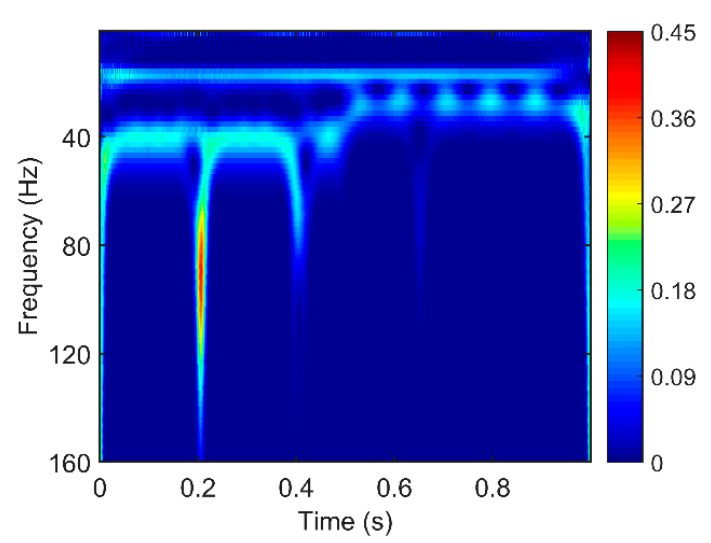

(a)

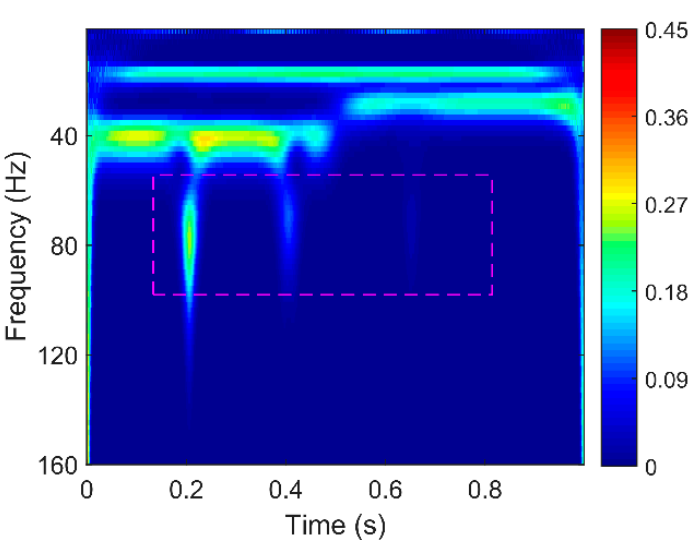

(b)

Figure 5. The instantaneous spectra of the synthetic signal obtained based on (a) the CWT/FWEO method; and (b) the VMD/CWT/FWEO method.

\section{Model Test}

The energy attenuation during seismic wave propagation is closely related to the lithology of the strata, the viscosity of liquid, and the dispersity of coupling between the solid and liquid phases. Korneev [48] successfully simulated the low frequency effects of fluid-saturated layers with the 1D diffusive-viscous wave equation theory, based on laboratory and field data. He explained that very low values of $Q$ (quality factor) are the result of internal diffusive losses caused by fluid flow. He [49] and Chen [50] separately extend the 1D diffusive-viscous wave equation to 2D and 3D dimensions. The application of theoretical models and practical data verified that the attenuation of the high-frequency components is greater than the low-frequency components. In this paper, we demonstrate the effectiveness of the VMD/CWT/FWEO method for hydrocarbon detection by means of numerical simulation. Firstly, we build a geological model and select the needed equivalent parameters $(\mathrm{Q}$ values, diffusive coefficients, and viscous coefficients) for the model, according to the seismic data and logging data of ZhongJiang Gas Field located in the Western Sichuan Basin, China (Figure 6a). Following this, we obtain a simulated seismic section (Figure 6a) by making use of the 2D diffusive-viscous wave equation [50]. The geological model has six formations and the parameters of each layer are shown in Table 1. The layer marked (4) is the gas-bearing layer and its thickness is $36 \mathrm{~m}$. The dominant frequency of the wavelet is $40 \mathrm{~Hz}$. The sampling frequency is $1000 \mathrm{~Hz}$. 


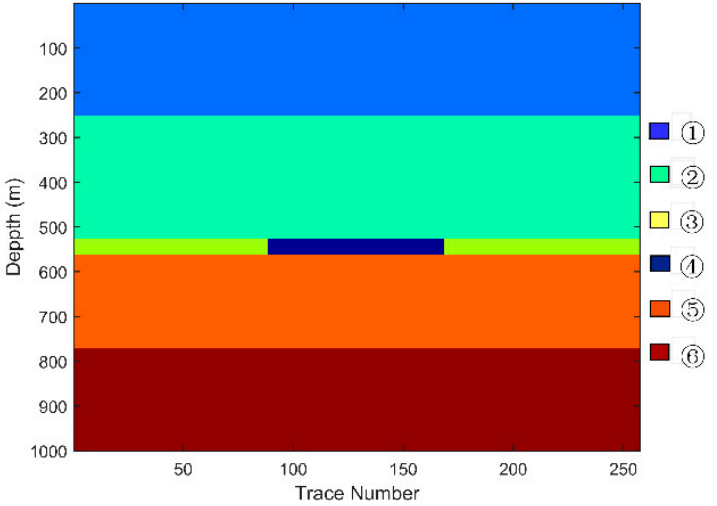

(a)

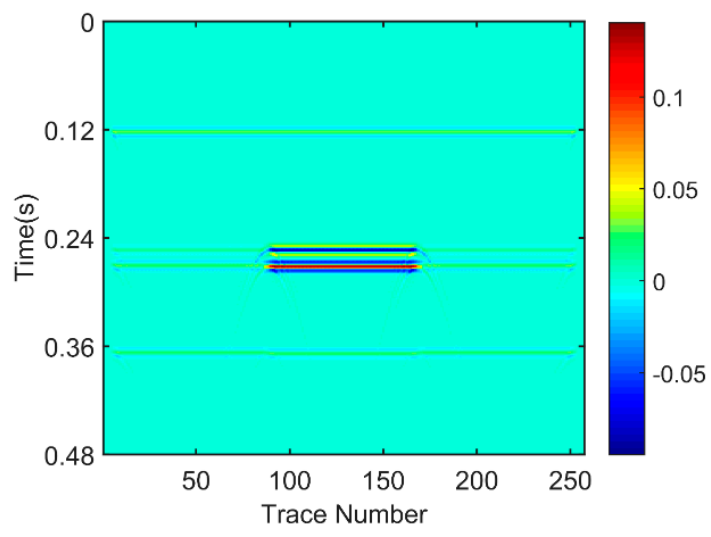

(b)

Figure 6. (a) Geological model; and (b) the simulated seismic section of the model.

Table 1. Main parameters for the geological model.

\begin{tabular}{|c|c|c|c|c|c|}
\hline Layer & $\begin{array}{l}\text { Velocity } \\
\left(\mathrm{m} \cdot \mathrm{s}^{-1}\right)\end{array}$ & $\begin{array}{l}\text { Diffusion Coefficient } \\
(\mathbf{H z})\end{array}$ & $\begin{array}{l}\text { Viscous Coefficient } \\
\left(\mathrm{m}^{2} \cdot \mathrm{s}^{-1}\right)\end{array}$ & $\begin{array}{l}\text { Density } \\
\left(\mathrm{g} \cdot \mathrm{cm}^{-3}\right)\end{array}$ & $\mathbf{Q}$ \\
\hline (1) & 4100 & 1.0 & 1.0 & 2.5 & 500 \\
\hline (2) & 4200 & 1.0 & 1.0 & 2.525 & 500 \\
\hline (3) & 4300 & 1.0 & 1.0 & 2.53 & 500 \\
\hline (4) & 4000 & 20.0 & 100 & 2.4 & 25 \\
\hline (5) & 4350 & 1.0 & 1.0 & 2.55 & 500 \\
\hline (6) & 4450 & 1.0 & 1.0 & 2.56 & 500 \\
\hline
\end{tabular}

\subsection{Hydrocarbon Detection in the Model}

After this, we use the VMD/CWT/FWEO method for time-frequency analysis and energy tracking in the simulated seismic section of the model. Following this, we extract the $44 \mathrm{~Hz}$ low-frequency section (Figure 7a) and the $54 \mathrm{~Hz}$ high-frequency section (Figure 7b). From Figure 7, we can obviously find a large amount of energy in the gas reservoir area in the low-frequency section (Figure 7a). However, in the high-frequency section (Figure 7b), the energy is weakened significantly. The energy attenuation from the low frequency section to the high-frequency section is mainly because of seismic waves travelling across a gas reservoir. Therefore, the detection results of the model prove that the $\mathrm{VMD} / \mathrm{CWT} / \mathrm{FWEO}$ method is effective for hydrocarbon detection.

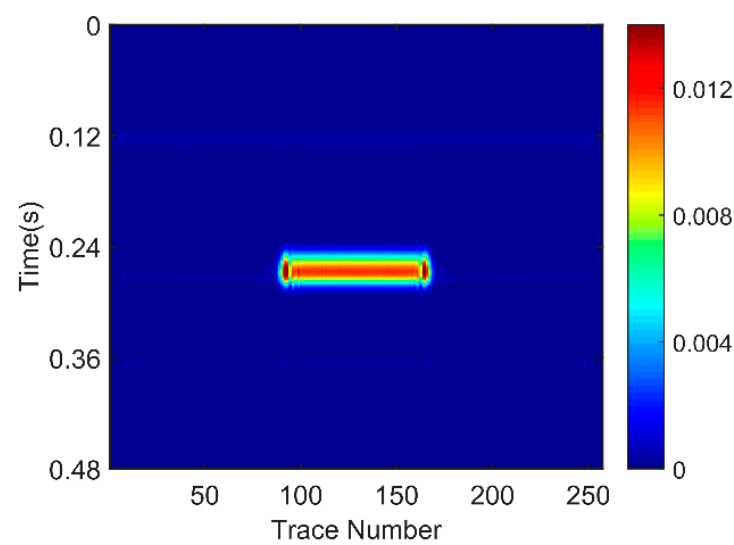

(a)

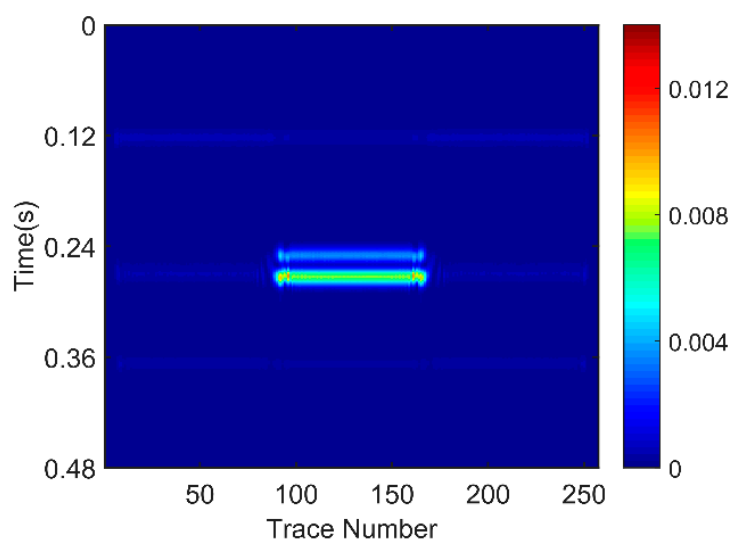

(b)

Figure 7. Constant frequency sections: (a) at $44 \mathrm{~Hz}$; and (b) at $54 \mathrm{~Hz}$. 


\subsection{Test Noise Robustness of the VMD/CWT/FWEO}

As we know, actual seismic data often contain various noises, which makes it very difficult to detect hydrocarbons. Thus, it is necessary to validate whether the VMD/CWT/FWEO method is robust to noise to some extent. Here, we produce three noise models by adding Gaussian white noises with different signal-to-noise ratios (SNR) to the simulated seismic section (Figure 6b). Noise model 1 is shown in Figure 8a with an SNR of $-2 \mathrm{~dB}$. Noise model 2 is shown in Figure 8d with a SNR of $-6 \mathrm{~dB}$. Noise model 3 is shown in Figure $8 \mathrm{~g}$ with a SNR of $-10 \mathrm{~dB}$. By applying the $\mathrm{VMD} / \mathrm{CWT} / \mathrm{FWEO}$ method to the three noise models, we respectively gain the constant frequency sections at a low frequency of $44 \mathrm{~Hz}$ and high frequency of $54 \mathrm{~Hz}$ in each noise model, as shown in Figure $8 b, c, e, f, h, i$. From these sections in Figure 8, we can easily observe that the detection results of noise models are similar to the result of the original section in Figure 6b. Strong energy that appeared at the low frequency section of each noise model was found to be weakened in the high-frequency section. Furthermore, in all the extracted constant frequency sections, the noise is nearly suppressed and has no effect on detection results.

Thus, we reasonably conclude that the proposed method in this paper is an effective tool for hydrocarbon detection and is of great robustness to noise. The method is worth generalizing and applying.

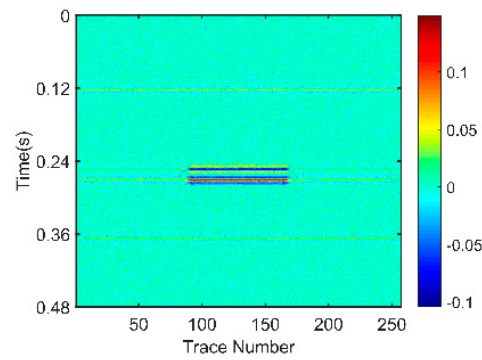

(a)

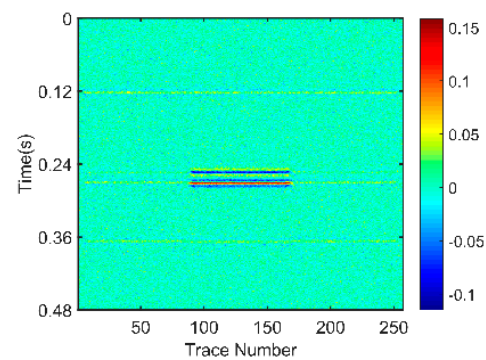

(d)

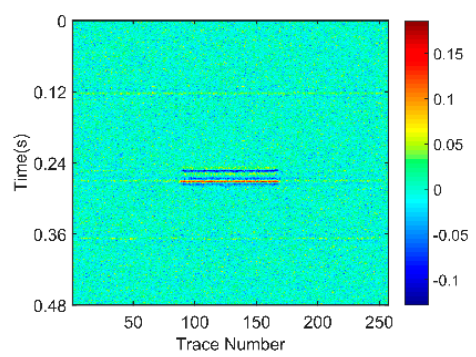

(g)

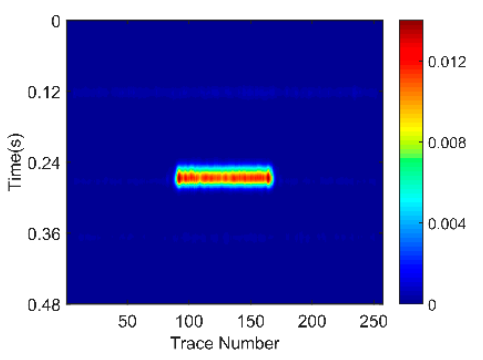

(b)

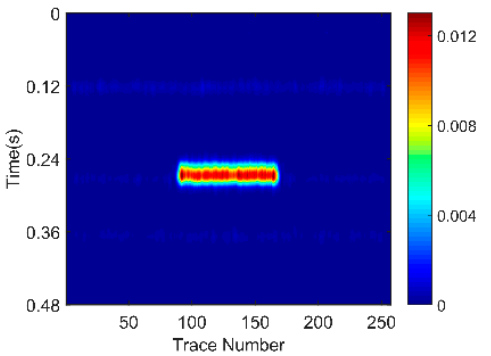

(e)

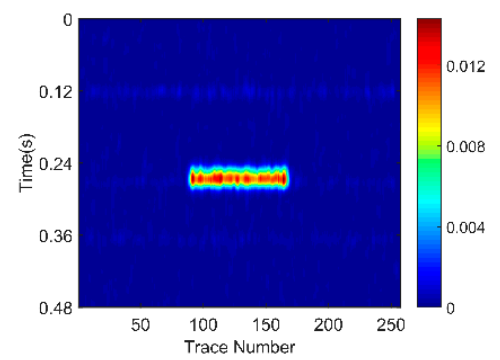

(h)

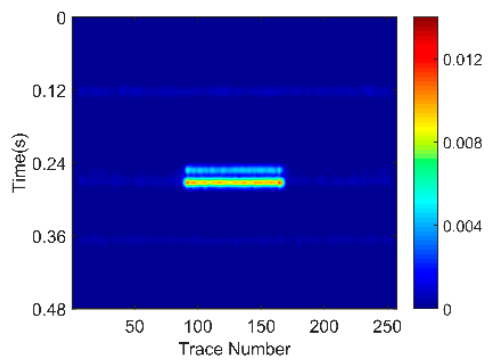

(c)

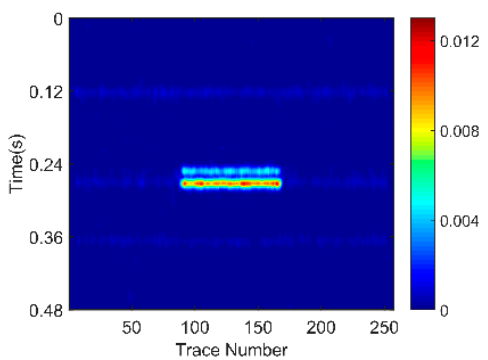

(f)

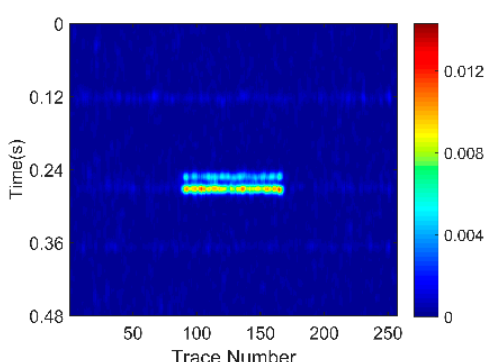

(i)

Figure 8. Noisy models and common frequency section of the noisy models: (a) noise model 1, SNR $=-2$; (b) low-frequency section of noise model 1; (c) high-frequency section of noise model 1; (d) noise model 2, SNR = -6; (e) low-frequency section of noise model 2; (f) high-frequency section of noise model 2; (g) noise model 3, SNR = -10; (h) low-frequency section of noise model 3; and (i) high-frequency section of noise model 3. 


\section{Seismic Data}

\subsection{Study Area Background}

In order to further validate the feasibility and practicality of the VMD/CWT/FWEO method for hydrocarbon detection in tight sandstone reservoirs, we adopt the field seismic data for analysis from the ZhongJiang Gas Field located in the western part of the Sichuan Basin, China. Furthermore, the gas field is mainly composed of tight sandstone reservoirs. In the field seismic data, a section intersects with two wells, as shown in Figure 9. The black curve at around $1 \mathrm{~ms}$ is a seismic horizon and the area within the black ellipse is a well-developed gas-bearing reservoir. The two black vertical lines denote two wells (Well A and Well B), respectively. Well A is a gas well and its open flow capacity is $2 \times 10^{4} \mathrm{~m}^{3} \mathrm{~d}^{-1}$. Well $\mathrm{B}$ is a dry well. The seismic section consists of 338 traces with 551 sampling points and a sampling interval of $2 \mathrm{~ms}$. Following this, the proposed method is applied to this section for hydrocarbon detection.

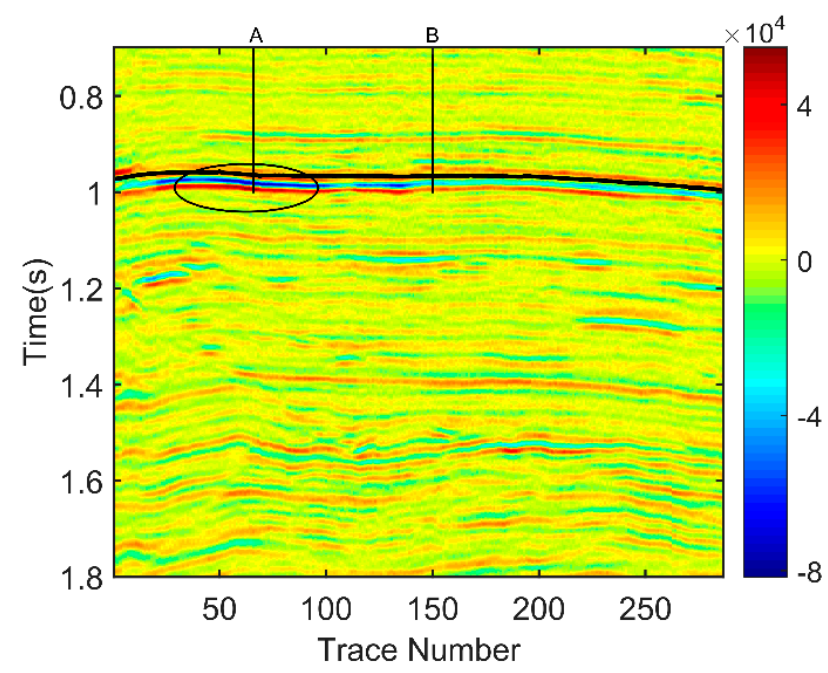

Figure 9. Field seismic data.

\subsection{Hydrocarbon Detection}

According to the running procedures of the VMD/CWT/FWEO method, we can extract constant frequency sections from the original section in Figure 9. Figure 10 depicts the constant frequency sections of $31 \mathrm{~Hz}$ (Figure 10a) and $43 \mathrm{~Hz}$ (Figure 10b). It is very clearly seen that a strong energy zone intersects with well A within the area labelled with red ellipse in the low frequency $(31 \mathrm{~Hz})$ section. In addition, the strong energy weakens in the high frequency $(43 \mathrm{~Hz})$ section. This is an obvious energy attenuation phenomena occurring between low-frequency and high-frequency sections, which illustrates that the area is a prolific gas-bearing reservoir. However, we cannot see the energy attenuation in the green area intersecting with well $\mathrm{B}$ between the two common frequency sections. Therefore, we can conclude that well B is not a gas well. The detection results coincide with the actual drilling data. Therefore, we can draw a conclusion that the VMD/CWT/FWEO method has the ability to effectively predict gas-bearing reservoirs in the tight sandstone reservoirs. 


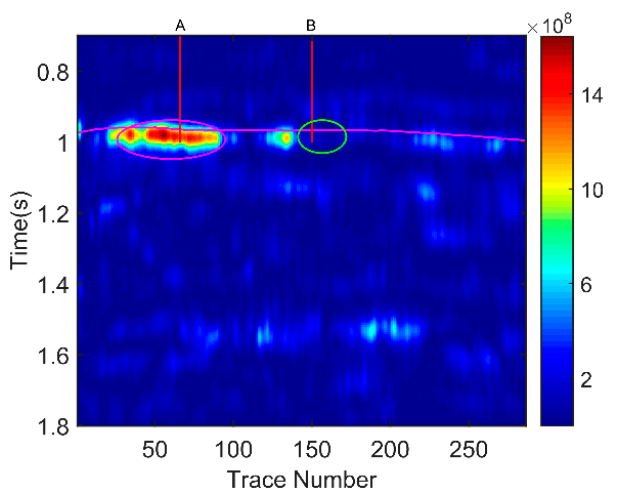

(a)

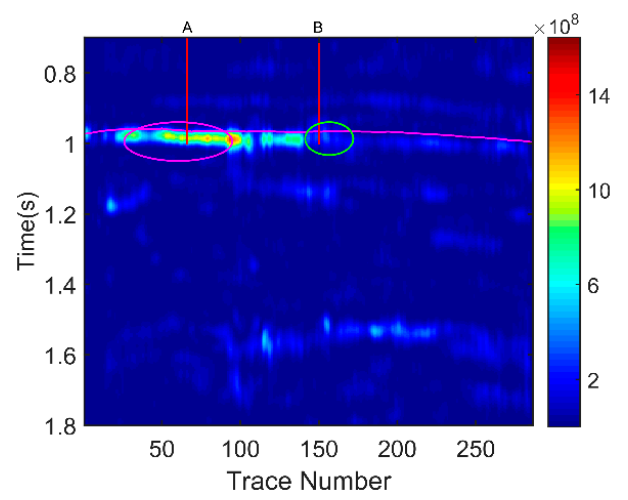

(b)

Figure 10. Constant frequency sections based on VMD/CWT/FWEO: (a) at $31 \mathrm{~Hz}$; and (b) at $43 \mathrm{~Hz}$.

\section{Conclusions}

In this paper, we have proposed a new hydrocarbon detection method in tight sandstone reservoirs using VMD, CWT and FWEO, which is named the VMD/CWT/FWEO method. Furthermore, the proposed method has a high time-frequency focus and resolution, so we can clearly find different frequency components of the signal in the time-frequency spectrum and instantaneous energy sections. Thus, we can easily detect hydrocarbons using the attenuation phenomenon between the low constant frequency section and the high constant frequency section extracted from the instantaneous energy spectrum. Decomposing a seismic signal using the VMD method before the CWT procedure not only improves the resolution of the time-frequency spectrum, but also avoids the loss of some frequency components caused by direct CWT analysis for seismic signals [22]. Therefore, the FWEO method is applied to track the instantaneous energy in the time-frequency spectrum, which can simultaneously obtain time and frequency localization information of abnormal energy. Compared with TK, the instantaneous energy values calculated by the FWEO method are more accurate, having explicit importance in physics without negative meaning. It is important that the VMD/CWT/FWEO is shown to have good anti-noise properties by testing noise models with different SNR. The model tests and practical application show that the proposed method can effectively detect hydrocarbons in tight sandstone gas reservoirs and is insensitive to noise. Therefore, the method is highly promising and has extensive applicative value in hydrocarbon detection in tight sandstone reservoirs. However, the method proposed in this paper for hydrocarbon detection requires the scale parameter of wavelet analysis to be set in advance.

Acknowledgments: The work presented in this paper has been supported by the National Natural Science Foundation of China (No. 41304111 and No. 41672325), National Key R\&D Program of China (No. 2017YFC0601505), key project of Science Technology Department of Sichuan Province (No. 2016JY0200), Natural Science project of Education Department of Sichuan Province (Nos. 14ZA0061, 16ZB0101, and 17ZA0030), the Sichuan Provincial Youth Science and Technology Innovative Research Group Fund (No. 2016TD0023), and the Cultivating Programme of Excellent Innovation Team of Chengdu University of Technology (No. KYTD201410).

Author Contributions: Hui Chen conducted all work in this paper. Dan Xu and Ying Hu conceived, designed, and performed the experiments. Ke Guo provided seismic data, some ideas and suggestions. Dan Xu and Xinyue Zhou analyzed the data. Dan Xu, Ying Hu, and Xinyue Zhou wrote the paper.

Conflicts of Interest: The authors declare no conflict of interest.

\section{References}

1. Holditch, S.A. Tight gas sands. J. Pet. Technol. 2006, 58, 86-93. [CrossRef]

2. Guo, Y.C.; Pang, X.Q. The critical buoyancy threshold for tight sandstone gas entrapment: Physical simulation, interpretation, and implications to the Upper Paleozoic Ordos Basin. J. Pet. Sci. Eng. 2017, 149, 88-97. [CrossRef] 
3. Vogelaar, B.; Smeulders, D. Exact expression for the effective acoustics of patchy-saturated rocks. Geophysics 2010, 75, N87-N96. [CrossRef]

4. Norris, A.N. Low-frequency dispersion and attenuation in partially saturated rocks. J. Acoust. Soc. Am. 1993, 94, 359-370. [CrossRef]

5. White, J.E. Computed seismic speeds and attenuation in rocks with partial gas saturation. Geophysics 2012, 40, 224. [CrossRef]

6. Johnson, D.L. Theory of frequency dependent acoustics in patchy-saturated porous media. J. Acoust. Soc. Am. 2001, 110, 682-694. [CrossRef]

7. Müller, T.M.; Gurevich, B. Wave-induced fluid flow in random porous media: Attenuation and dispersion of elastic waves. J. Acoust. Soc. Am. 2010, 117, 2732-2741. [CrossRef]

8. Dvorkin, J.; Nur, A. Dynamic poroelasticity; a unified model with the squirt and the Biot mechanisms. Geophysics 1993, 58, 524-533. [CrossRef]

9. Xiong, X.J.; He, X.L. High-precision frequency attenuation analysis and its application. Appl. Geophys. 2011, 8, 337-343. [CrossRef]

10. Pride, S.R.; Berryman, J.G. Seismic attenuation due to wave-induced flow. J. Geophys. Res. Atmos. 2003, 109, B01201. [CrossRef]

11. Duchesne, M.J.; Halliday, E.J. Analyzing seismic imagery in the time-amplitude and time-frequency domains to determine fluid nature and migration pathways: A case study from the Queen Charlotte Basin, offshore British Columbia. J. Appl. Geophys. 2011, 73, 111-120. [CrossRef]

12. Cadoret, T.; Mavko, G. Fluid distribution effect on sonic attenuation in partially saturated limestones. Geophysics 1998, 63, 154-160. [CrossRef]

13. Klimentos, T. Attenuation of P-and S-waves as a method of distinguishing gas and condensate from oil and water. Geophysics 1995, 60, 447-458. [CrossRef]

14. Winkler, U.K.; Stuckmann, M. Glycogen, hyaluronate, and some other polysaccharides greatly enhance the formation of exolipase by Serratia marcescens. J. Bacteriol. 1979, 138, 663-670. [PubMed]

15. Taner, M.T.; Koehler, F. Complex seismic trace analysis. Geophysics 1979, 44, 1041-1063. [CrossRef]

16. Xue, Y.J.; Cao, J.X. EMD and Teager-Kaiser energy applied to hydrocarbon detection in a carbonate reservoir. Geophys. J. Int. 2014, 199, 277-291. [CrossRef]

17. De Matos, M.C.; Marfurt, K.J. Wavelet transform Teager-Kaiser energy applied to a carbonate field in Brazil. Lead. Edge 2009, 28, 708-713. [CrossRef]

18. Castagna, J.P.; Sun, S. Instantaneous spectral analysis: Detection of low-frequency shadows associated with hydrocarbons. Lead. Edge 2003, 22, 120-127. [CrossRef]

19. Liu, W.; Cao, S.Y. Spectral Decomposition for Hydrocarbon Detection Based on VMD and Teager-Kaiser Energy. IEEE Geosci. Remote Sens. Lett. 2017, 14, 539-543. [CrossRef]

20. Xue, Y.J.; Cao, J.X. A comparative study on hydrocarbon detection using three EMD-based time-frequency analysis methods. J. Appl. Geophys. 2013, 89, 108-115. [CrossRef]

21. Goloshubin, G.M. Seismic low-frequency effects from fluid-saturated reservoir. In SEG Technical Program Expanded Abstracts 2000; Society of Exploration Geophysicists: Tulsa, OK, USA, 2000; pp. 1671-1674.

22. Xue, Y.J.; Cao, J.X. Application of the empirical mode decomposition and wavelet transform to seismic reflection frequency attenuation analysis. J. Pet. Sci. Eng. 2014, 122, 360-370. [CrossRef]

23. Partyka, G.; Gridley, J. Interpretational applications of spectral decomposition in reservoir characterization. Lead. Edge 1999, 18, 353-360. [CrossRef]

24. Huang, Y.P.; Geng, J.H. Seismic attribute extraction based on HHT and its application in a marine carbonate area. Appl. Geophys. 2011, 8, 125-133. [CrossRef]

25. Liu, C.C.; Chen, B.S. Reassigned wavelet spectral decomposition and its application in hydrocarbon detection. In SEG Technical Program Expanded Abstracts 2013; Society of Exploration Geophysicists: Tulsa, OK, USA, 2013; pp. 2611-2615.

26. Wang, L.; Gao, J. Hydrocarbon detection using adaptively selected spectrum attenuation. J. Appl. Geophys. 2014, 105, 59-66. [CrossRef]

27. Li, F.Y.; Zhou, H.L. Seismic Spectral Attributes of Apparent Attenuation: Part 2-Application. In SEG Technical Program Expanded Abstracts 2015; Society of Exploration Geophysicists: Tulsa, OK, USA, 2015; pp. 2682-2687.

28. Huang, N.E.; Shen, Z. The empirical mode decomposition and the Hilbert spectrum for nonlinear and non-stationary time series analysis. Proc. R. Soc. A Math. Phys. Eng. Sci. 1998, 454, 903-995. [CrossRef] 
29. Han, J.J.; van der Baan, M. Empirical mode decomposition for seismic time-frequency analysis. Geophysics 2013, 78, O9-O19. [CrossRef]

30. Liu, W.; Cao, S.Y. Seismic time-frequency analysis via empirical wavelet transform. IEEE Geosci. Remote Sens. Lett. 2016, 13, 28-32. [CrossRef]

31. Gilles, J. Empirical Wavelet Transform. IEEE Trans. Signal Process. 2013, 61, 3999-4010. [CrossRef]

32. Huang, N.E.; Wu, Z.H. A review on Hilbert-Huang transform: Method and its applications to geophysical studies. Rev. Geophys. 2008, 46, 1-23. [CrossRef]

33. Rato, R.; Ortigueira, M. On the HHT, its problems, and some solutions. Mech. Sys. Signal Process. 2008, 22, 1374-1394. [CrossRef]

34. Torres, M.E.; Colominas, M.A. A complete ensemble empirical mode decomposition with adaptive noise. In Proceedings of the 2011 IEEE International Conference on Acoustics, Speech and Signal Processing (ICASSP), Prague, Czech Republic, 22-27 May 2011; pp. 4144-4147.

35. Xue, Y.J.; Cao, J.X. Application of the Variational-Mode Decomposition for Seismic Time-frequency Analysis. IEEE J. Sel. Top. Appl. Earth Obs. Remote Sens. 2016, 9, 3821-3831. [CrossRef]

36. Liu, W.; Cao, S.Y. Applications of variational mode decomposition in seismic time-frequency analysis. Geophysics 2016, 81, V365-V378. [CrossRef]

37. Dragomiretskiy, K.; Zosso, D. Variational mode decomposition. IEEE Trans. Signal Process. 2014, 62, 531-544. [CrossRef]

38. Chandra, N.H.; Sekhar, A. Fault detection in rotor bearing systems using time frequency techniques. Mech. Syst. Signal Process. 2016, 72, 105-133. [CrossRef]

39. De Matos, M.C.; Marfurt, K.J. Brazilian deep water carbonate reservoir study using the wavelet transform Teager-Kaiser energy. In Proceedings of the SEG Annual Meeting, Las Vegas, NV, USA, 9-14 November 2008; pp. 1516-1520.

40. De Matos, M.C.; Johann, P.R. Revealing geological features through seismic attributes extracted from the wavelet-transform Teager-Kaiser energy. In Proceedings of the SEG Annual Meeting, San Antonio, TX, USA, 23-28 September 2007; pp. 1442-1446.

41. Kaiser, J.F. On a simple algorithm to calculate the 'energy' of a signal. In Proceedings of the International Conference on Acoustics, Speech and Signal Processing (ICASSP), Albuquerque Convention Center, Albuquerque, New Mexico, NM, USA, 3-6 April 1990; pp. 381-384.

42. O'Toole, J.M.; Temko, A. Assessing instantaneous energy in the EEG: A non-negative, frequency-weighted energy operator. In Proceedings of the Engineering in Medicine and Biology Society (EMBC), Chicago, IL, USA, 26-30 August 2014; pp. 3288-3291.

43. Imaouchen, Y.; Kedadouche, M. A Frequency-Weighted Energy Operator and complementary ensemble empirical mode decomposition for bearing fault detection. Mech. Sys. Signal Process. 2017, 82, 103-116. [CrossRef]

44. Bertsekas, D.P. Multiplier methods: A survey. Automatica 1976, 12, 133-145. [CrossRef]

45. Hestenes, M.R. Multiplier and gradient methods. J. Optim. Theory Appl. 1969, 4, 303-320. [CrossRef]

46. Daubechies, I. Ten Lectures on Wavelets; Society for Industrial and Applied Mathematics: Philadelphia, PA, USA, 1992; pp. 38-44.

47. Xiao, M.L.; Ye, L.Y. Application of continuous wavelet transform to seismic response analysis. World Inf. Earthq. Eng. 2001, 17, 79-83.

48. Korneev, V.A.; Goloshubin, G.M. Seismic low-frequency effects in monitoring fluid-saturated reservoirs. Geophysics 2004, 69, 522-532. [CrossRef]

49. He, Z.H.; Xiong, X.J. Numerical simulation of seismic low-frequency shadows and its application. Appl. Geophys. 2008, 5, 301-306. [CrossRef]

50. Chen, X.H.; He, Z.H. Numeric simulation and detection of low frequency Shadow. Oil Geophys. Prospect. 2009, 44, 298-303.

(C) 2017 by the authors. Licensee MDPI, Basel, Switzerland. This article is an open access article distributed under the terms and conditions of the Creative Commons Attribution (CC BY) license (http:/ / creativecommons.org/licenses/by/4.0/). 